\title{
A case report of fatal hepatic portal venous gas after transcatheter arterial chemoembolization in a patient with hepatocellular carcinoma
}

\author{
Lichao Xu", Ying Wang ${ }^{\#}$, Wentao Li \\ Department of Interventional Radiology, Fudan University Shanghai Cancer Center, Shanghai, China \\ \#These authors contributed equally to this work. \\ Correspondence to: Wentao Li. Department of Interventional Radiology, Fudan University Shanghai Cancer Center, 270 Dong An Road, Xuhui, \\ Shanghai 200032, China. Email: liwentao98@126.com.
}

\begin{abstract}
Hepatic portal venous gas (HPVG) refers to the imaging signs formed by the abnormal accumulation of abnormal gas in the portal veins and the branches of the inner veins of the liver due to various reasons, and is usually associated with poor prognosis. HPVG is mostly caused by abdominal diseases. Herein, we reported a patient with hepatocellular carcinoma developed HPVG following transcatheter arterial chemoembolization (TACE). This patient was identified as Barcelona Clinic Liver Cancer (BCLC) stage B, and treated by five times of TACE. Gelfoam sponge was used as embolic agent in the first and last TACE treatment. After the first TACE, pneumonia occurred in the right lung, and pneumorachis with iodine oil deposition occurred in the tumor lesion in the right lobe of the liver, which was considered as hepatic abscess. After three more TACE, enhanced MRI showed significant necrosis and reduction of the lesions. After the fifth TACE, the enhancing computed tomography (CT) showed a large amount of gas in the lesions and in the portal veins, which was considered as HPVG. Although after active treatment, the patient died soon. To our knowledge, this is the first case report of a patient with hepatocellular carcinoma developed HPVG following TACE with gelfoam sponge as embolic agent.
\end{abstract}

Keywords: Case report; hepatic portal venous gas (HPVG); hepatocellular carcinoma; transcatheter arterial chemoembolization (TACE); gelfoam sponge

Submitted Aug 24, 2021. Accepted for publication Nov 04, 2021.

doi: $10.21037 /$ tcr-21-1721

View this article at: https://dx.doi.org/10.21037/tcr-21-1721

\section{Introduction}

Primary hepatocellular carcinoma has a high incidence and mortality rate in China (1). The treatment of hepatocellular carcinoma is limited, and although new treatment methods are emerging, the clinical benefits are limited (2). Transcatheter arterial chemoembolization (TACE) is an important therapy in the treatment of hepatocellular carcinoma. Gelfoam sponge has been commonly used as an embolic agent in clinical practice.

Hepatic portal venous gas (HPVG) refers to the imaging signs formed by the abnormal accumulation of gas in the door veins and the branches of the inner veins of the liver due to various reasons, and is a rare imaging sign $(3,4)$. HPVG was reported to occur commonly when intestinal isoemia and necrosis occur, and sepsis, abdominal infections and other non-digestive tract lesions also cause HPVG (5). Here, we reported a patient with hepatocellular carcinoma developed HPVG following TACE, resulting in a poor prognosis. We present the following article in accordance with the CARE reporting checklist (available at https:// dx.doi.org/10.21037/tcr-21-1721).

^ ORCID: 0000-0001-7475-0208. 

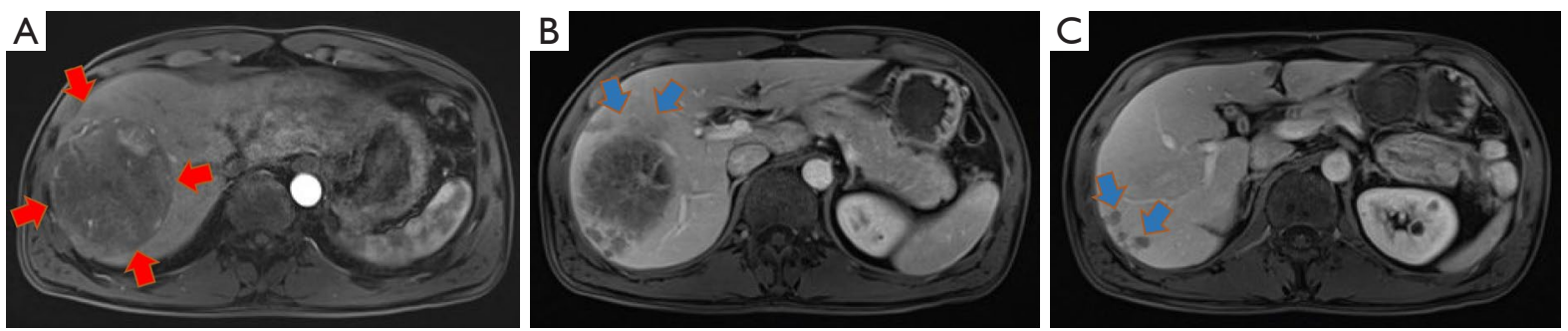

Figure 1 Enhanced MRI at baseline. (A) A mass $(9.8 \mathrm{~cm} \times 7.9 \mathrm{~cm})$ in the right hepatic lobe (red arrows); (B,C) small lesions around the mass (blue arrows). MRI, magnetic resonance imaging.
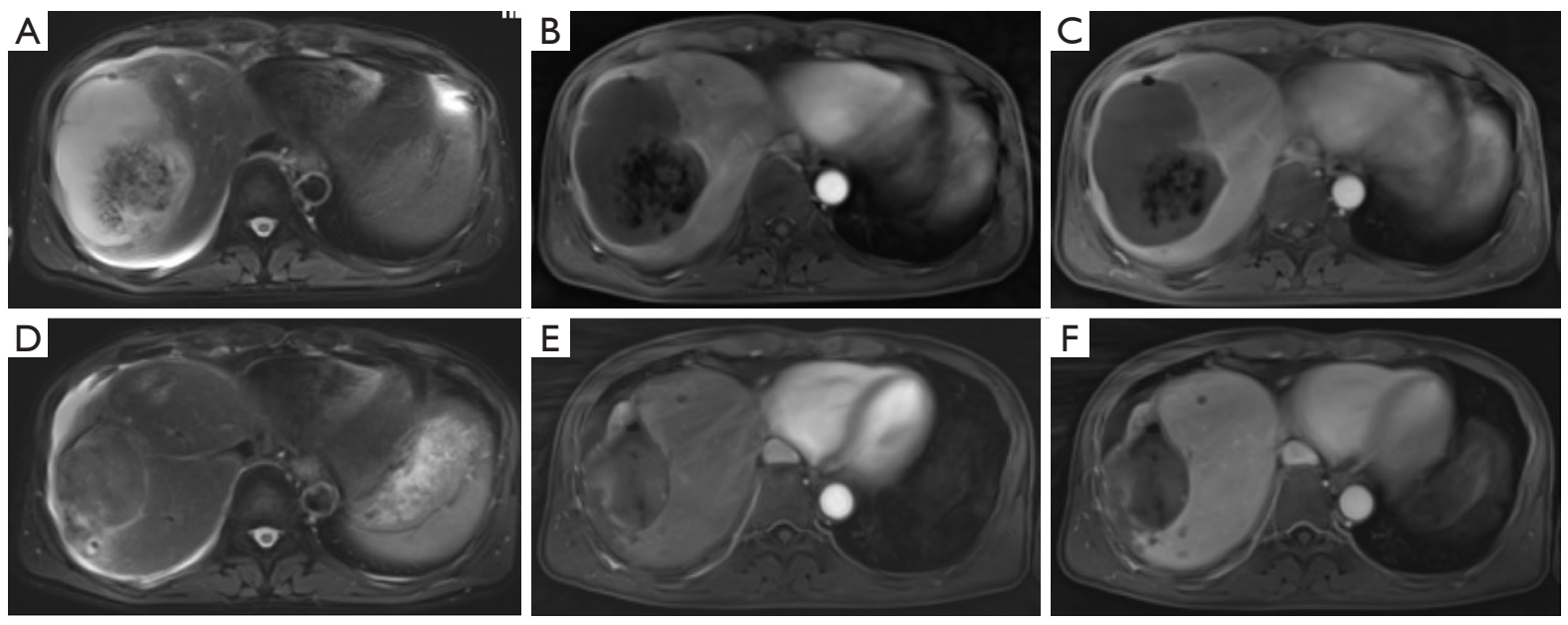

Figure 2 The abdomen enhanced MRI showed the liver lesion was well controlled. (A,B,C) Significant necrosis of the lesions after the first TACE; (D,E,F) significant necrosis and reduction of the lesions after the fourth TACE. TACE, transcatheter arterial chemoembolization.

\section{Case presentation}

A 44-year-old male with a history of hepatitis B was diagnosed with primary hepatocellular carcinoma. The patient previously suffered from chronic viral hepatitis B without cirrhosis. Multiphasic contrast-enhanced Magnetic Resonance Imaging (MRI) showed a $9.8 \mathrm{~cm} \times 7.9 \mathrm{~cm}$ mass in the right hepatic lobe with several small lesions and arterial hypervascularity (Figure 1). Alpha-fetoprotein (AFP) was greater than $3,630 \mathrm{ng} / \mathrm{mL}$. According to European Association for the Study of the Liver (EASL) Clinical Practice Guidelines (6), this patient was identified as Barcelona Clinic Liver Cancer (BCLC) stage B, and transarterial chemoembolization is the standard treatment.

May 8, 2018, the patient was treated by the first TACE (30 mg THP loaded by 100-300 $\mu \mathrm{m}$ drug micro-ball, $8 \mathrm{~mL}$ iodized oil, $350 \mu \mathrm{m}$ gelfoam sponge particles). After one day following TACE, the patient developed a fever, up to a maximum of $39.3^{\circ} \mathrm{C}$, and developed severe liver damage. The laboratory test showed that white blood cell (WBC) was $8.5 \times 10^{9} / \mathrm{L}$ (normal range: $4 \times 10^{9}-10 \times 10^{9} / \mathrm{L}$ ), neutrophil granulocyte (GRAN) $91.4 \%$ (normal range: 50-70\%), alanine aminotransferase (ALT) 2.6 upper limit of normal value (ULN) (normal range: 0-40 U/L), aspartate aminotransferase (AST) 7.9 ULN (normal range: 0-40 U/L), and total bilirubin (TBIL) 1.9 ULN (normal range: $1.71-17.1 \mu \mathrm{mol} / \mathrm{L})$. Then he received treatment for liver preservation and other treatments. One month after TACE, ALT, AST and TBIL returned to normal range, while $\mathrm{WBC}$ was $9.2 \times 10^{9} / \mathrm{L}$ and GRAN was $82.7 \%$. At the same time, the abdominal enhancement MRI showed significant necrosis of the lesions (Figure 2), with AFP dropping to $1,708 \mathrm{ng} / \mathrm{mL}$. However, from June 15, 2018, the patient experienced repeated fever and cough, even after anti-infection treatment. The body temperature reached a maximum of $39^{\circ} \mathrm{C}$. The Chest computed tomography (CT) showed pneumonia occurred in the right lung, and 

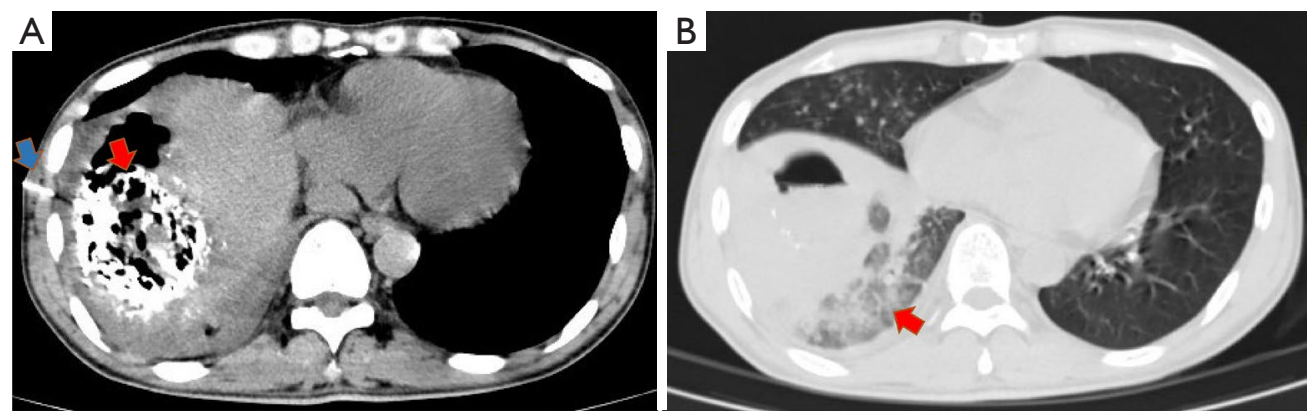

Figure 3 The CT showed hepatic abscess and pneumonia after the first TACE. (A) Pneumorachis with iodine oil deposition occurred in the tumor lesion in the right lobe of the liver (red arrow) and pigtail catheter drainage (blue arrow); (B) pneumonia occurred in the right lung (red arrow). CT, computed tomography; TACE, transcatheter arterial chemoembolization.
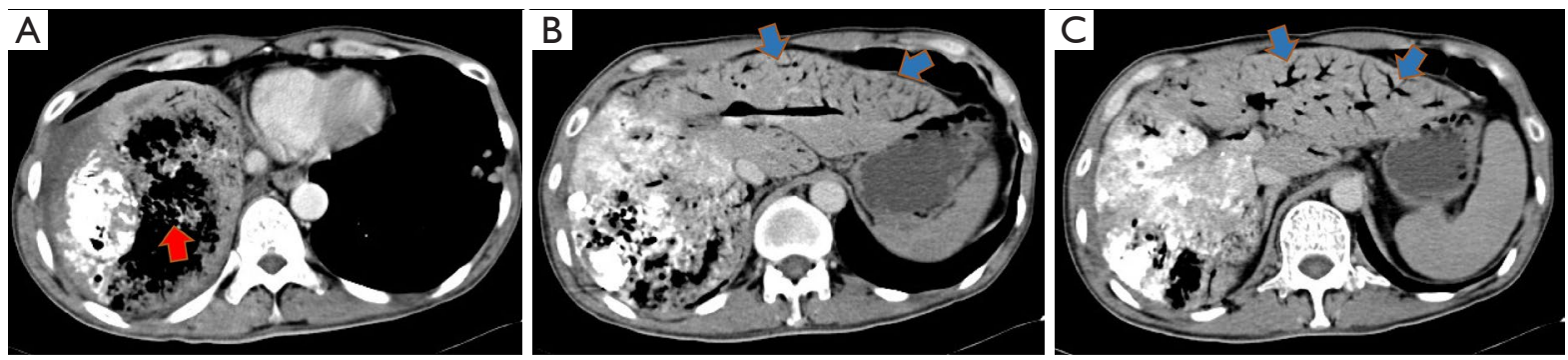

Figure 4 The abdomen enhanced CT showed HPVG after the fifth TACE. The CT showed a large amount of gas in the lesions (A; red arrow) and in the portal veins (B,C; blue arrows). CT, computed tomography; HPVG, hepatic portal venous gas; TACE, transcatheter arterial chemoembolization.

pneumorachis with iodine oil deposition occurred in the tumor lesion in the right lobe of the liver, which was considered as hepatic abscess (Figure 3). We continued the anti-infection treatment (Tazobactam Sodium/Piperacillin Sodium for Injectio, $4.5 \mathrm{~g}$ q $8 \mathrm{~h}$ for 7 days) and pigtail catheter drainage was administered, but the bacteria culture was negative. On July 31, 2018, a significant reduction in the original lesion in the right lobe of the liver was observed by enhanced MRI, but the number of new lesions in the liver increased and the AFP rose to more than $3,630 \mathrm{ng} / \mathrm{mL}$.

From August to November 2018, the patient received three more TACE $(60 \mathrm{mg}$ THP plus $20 \mathrm{~mL}, 8 \mathrm{~mL}, 7 \mathrm{~mL}$ iodized oil, respectively). November 28, 2018, enhanced MRI showed significant necrosis and reduction of the lesions (Figure 2). November 30, the patient received the fifth TACE (60 mg THP plus $4 \mathrm{~mL}$ iodized oil plus $350 \mu \mathrm{m}$ gelatin sponge particles). Fever began the day after TACE, reaching $39.6^{\circ} \mathrm{C}$. The laboratory test showed that WBC was $14.1 \times 10^{9} / \mathrm{L}$, GRAN $98.5 \%$, ALT $163 \mathrm{U} / \mathrm{L}$, AST 249 U/L, TBIL $73 \mu \mathrm{mol} / \mathrm{L}$. Then the patient received anti- infection and liver protection treatment. However, three days after the fifth TACE, the patient suddenly fainted and had difficulty breathing. The enhancing CT showed a large amount of gas in the lesions and in the portal veins (Figure 4). The bacteria cultured showed that Streptococcus anginosus from hepatic puncture tube drainage was positive. Although after active treatment, the patient died two hours after entering the intensive care unit (ICU). All procedures performed in studies involving human participants were in accordance with the ethical standards of the institutional committee and with the Helsinki Declaration (as revised in 2013). Written informed consent was obtained from the patient's next of kin for publication of this case report and accompanying images. A copy of the written consent is available for review by the editorial office of this journal.

\section{Discussion}

Herein, we reported a patient with hepatocellular carcinoma developed HPVG following TACE, which was rarely reported before. 
HPVG is a severe radiological symptom in which gas accumulates in the portal venous system. It was first reported in children by Wolfe and Evans in 1955 and in adults by Susman and Senturia in 1960 (7,8). Traditionally, HPVG was considered as being an indicator of poor prognosis and as being associated with a particularly high mortality rate up to $75 \%$ (3). With the development of highly advanced imaging techniques, it is gradually recognized that HPVG is not always a fatal sign, but maybe an iatrogenic and non-iatrogenic manifestation of severe abdominal diseases (5). According to literature retrieval, the causes for HPVG include bowel ischemia, severe enteritis/intestinal perforation/intestinal fistula, intestinal obstruction, celiac infection, gastric disease, abdominal trauma and other rare causes $(5,9,10)$. With regard to rare causes, HPVG was reported in a patient with hepatic artery embolization because of active bleeding after abdominal trauma (11). Recently, Bao reported a case of HPVG after radical cholangiocarcinectomy (12).

The diagnosis of HPVG is usually made by plain abdominal radiography, sonography, color Doppler flow imaging, or CT scan (13). Of these, CT scan can effectively detect HPVG and even can reveal potential pathology. CT scan shows that HPVG is typically related to the peripheral gas lucencies, and the distribution of these gases is affected by blood flow into the liver. Of note, HPVG is needed to be distinguished from pneumobilia, which is typically associated with central location and affected by the biliary anatomy and bile flow. In addition, CT scans can reveal gas in the intestinal wall (pneumatosis intestinalis) and external veins or splanchnic vasculature. One study showed that HPVG related to intestinal necrosis requires surgery, and ascites, peritoneal irritation signs, and shock were significantly related to poor prognosis (14).

In this case, iodine oil deposition occurred in the right lobe of the liver following the first TACE, which was considered as liver abscess. According to literatures, the incidence of liver abscess after TACE for HCC was $0.0-7 \%(15)$. Liver abscess is reported as a common adverse reaction following TACE with mortality rates ranging from $13.3 \%$ to $50 \%$ (16). The cause of liver abscess after TACE remains unclear and is complex. Several mechanisms may contribute to the formation liver abscess after TACE: liquefied necrosis caused by tumor tissue embolization and liver dysfunction induced by chemotherapy, decline systemic immunity and thereby induce abscess formation. In general, small diameter of gelfoam particles (such as 100-300 $\mu \mathrm{m}$ ), large tumor size, uncontrolled diabetes, cholecostolithiasis, type II biliary abnormalities, concomitant portal vein tumor thrombus, and multiple sessions of TACE, and declined immunity, are considered as risk factors for liver abscess after TACE (16-19). Small diameter of gelfoam particles was considered as a risk factor for liver abscess formation after TACE. Besides, it is reported that gelfoam particles were applied in a case series of liver abscess formation after TACE for liver tumors (20), which indicating that the use of gelfoam and lipiodol may increase the risk of liver abscess formation for patients with portal vein tumor thrombus after TACE. Similar results were also observed in another study (19). These phenomena may be explained by the semifluid nature of iodized oil, which would block the hepatic portal vein together with the gelatin particulate, and produce a "subsegmentectomy like effect", which is the coexistence of massive tumor destruction and adjacent liver parenchyma atrophy. So the authors considered that the use of gelfoam particles in addition to iodized oil would increase the risk of developing liver abscess when the above risk factors existed. So the operator should pay attention to the occurrence of liver abscess even the more serious complication, such as HPVG, when use iodized oil and gelfoam particles simultaneously.

After the other three TACE, the liver lesion was well controlled. But after the fifth TACE with gelatin sponge embolism, the patient developed chest tightness, breathing difficulties, and abdominal pain. The enhanced CT found a large amount of gas in the lesions and in the portal veins, and no intestinal ischemia happened. It is speculated that the use of gelatin sponge embolism caused liver abscesses, and then gas-producing bacteria spread to the intestinal mucous membranes and small veins, resulting in accumulation of gas in the portal veins, which was supported by positive Streptococcus anginosus from hepatic puncture tube drainage. Although the patient immediately received active anti-infection, liver preservation and other treatment, he still died soon after. Therefore, it should be alert to the occurrence of fatal HPVG when patients with hepatocellular carcinoma treated by TACE with gelatin sponge embolism developed liver abscesses.

HPVG is not just a special radiological feature, it can be fatal. There is some speculation about the cause of HPVGrelated death. Microbubbles may occur before HPVG is radiologically visible. These microbubbles could cause protein denaturation in the portal sinusoids, which slows down the portal flow. As microbubbles accumulate, portal sinusoids may be blocked by protein gels and bubbles. Sufficient microbubbles make HPVG radiologically visible 
and partially or completely eliminate blood flow in the portal veins. As the venous return is damaged, necrosis containing bacteria and toxins would become increasingly engorged and transperitoneal exudation would increase. Bacterial infections and toxins will result in septicaemia and poor prognosis. So the authors thought the amount of gas, the speed of gas generation, and the severity of associated infection maybe the main reasons which decided whether the HPVG was fetal or not. It was possible that a large amount and rapid generation HPVG, with uncontrolled infection indicated the patient's death. An alternative hypothesis reported by Carroll et al. (21), that gas within embolized, uninfected tumors may come from either carbon dioxide produced through anaerobic reticulocyte metabolism or oxygen from oxyhemoglobin.

\section{Acknowledgments}

Funding: None.

\section{Footnote}

Reporting Checklist: The authors have completed the CARE reporting checklist. Available at https://dx.doi. org/10.21037/tcr-21-1721

Conflicts of Interest: All authors have completed the ICMJE uniform disclosure form (available at https://dx.doi. org/10.21037/tcr-21-1721). The authors have no conflicts of interest to declare.

Ethical Statement: The authors are accountable for all aspects of the work in ensuring that questions related to the accuracy or integrity of any part of the work are appropriately investigated and resolved. All procedures performed in studies involving human participants were in accordance with the ethical standards of the institutional committee and with the Helsinki Declaration (as revised in 2013). Written informed consent was obtained from the patient's next of kin for publication of this case report and accompanying images. A copy of the written consent is available for review by the editorial office of this journal.

Open Access Statement: This is an Open Access article distributed in accordance with the Creative Commons Attribution-NonCommercial-NoDerivs 4.0 International License (CC BY-NC-ND 4.0), which permits the noncommercial replication and distribution of the article with the strict proviso that no changes or edits are made and the original work is properly cited (including links to both the formal publication through the relevant DOI and the license). See: https://creativecommons.org/licenses/by-nc-nd/4.0/.

\section{References}

1. Zhang S, Sun K, Zheng R, Zeng H, Wang S, Chen R, et al. Cancer incidence and mortality in China, 2015. Journal of the National Cancer Center. 2020.

2. Finn RS, Zhu AX. Evolution of Systemic Therapy for Hepatocellular Carcinoma. Hepatology 2021;73 Suppl 1:150-7.

3. Nelson AL, Millington TM, Sahani D, et al. Hepatic portal venous gas: the ABCs of management. Arch Surg 2009;144:575-81; discussion 581 .

4. Abboud B, El Hachem J, Yazbeck T, et al. Hepatic portal venous gas: physiopathology, etiology, prognosis and treatment. World J Gastroenterol 2009;15:3585-90.

5. Daneshmand A, Parys S, Rao S, et al. Portal venous gas: different aetiologies and their respective outcomes. ANZ J Surg 2020;90:767-71.

6. European Association for the Study of the Liver. Electronic address: easloffice@easloffice.eu; European Association for the Study of the Liver. EASL Clinical Practice Guidelines: Management of hepatocellular carcinoma. J Hepatol 2018;69:182-236.

7. Wolfe JN, Evans WA. Gas in the portal veins of the liver in infants; a roentgenographic demonstration with postmortem anatomical correlation. Am J Roentgenol Radium Ther Nucl Med 1955;74:486-8.

8. Susman N, Senturia HR. Gas embolization of the portal venous system. Am J Roentgenol Radium Ther Nucl Med 1960;83:847-50.

9. Mourad FH, Leong RW. Gas In The Hepatic Portal Venous System Associated With Ischemic Colitis. Am J Gastroenterol 2018;113:1280.

10. Murzi M, Gordillo J, Oblitas E, et al. Spontaneous hepatic portal venous gas in a patient with ulcerative colitis. A case report and a review. Gastroenterol Hepatol 2020;43:22-5.

11. McCarthy P, Adam A, Jackson J, et al. Computed tomography demonstration of portal venous gas after hepatic artery embolization. Br J Radiol 1990;63:647-8.

12. Bao LX, Hu X, Guo WD. Hepatic portal venous gas after radical cholangiocarcinectomy. Quant Imaging Med Surg 2020;10:2376-8.

13. Shah PA, Cunningham SC, Morgan TA, et al. Hepatic gas: widening spectrum of causes detected at CT and US in the 
interventional era. Radiographics 2011;31:1403-13.

14. Gonda M, Osuga T, Ikura Y, et al. Optimal treatment strategies for hepatic portal venous gas: A retrospective assessment. World J Gastroenterol 2020;26:1628-37.

15. Sun Z, Li G, Ai X, et al. Hepatic and biliary damage after transarterial chemoembolization for malignant hepatic tumors: incidence, diagnosis, treatment, outcome and mechanism. Crit Rev Oncol Hematol 2011;79:164-74.

16. Li JH, Yao RR, Shen HJ, et al. Clostridium perfringens infection after transarterial chemoembolization for large hepatocellular carcinoma. World J Gastroenterol 2015;21:4397-401.

17. Sun W, Xu F, Li X, et al. A Case Series of Liver Abscess Formation after Transcatheter Arterial Chemoembolization for Hepatic Tumors. Chin Med J

Cite this article as: $\mathrm{Xu} \mathrm{L}$, Wang $\mathrm{Y}, \mathrm{Li} \mathrm{W}$. A case report of fatal hepatic portal venous gas after transcatheter arterial chemoembolization in a patient with hepatocellular carcinoma. Transl Cancer Res 2021;10(12):5437-5442. doi: 10.21037/tcr$21-1721$
(Engl) 2017;130:1314-9.

18. Kim W, Clark TW, Baum RA, et al. Risk factors for liver abscess formation after hepatic chemoembolization. J Vasc Interv Radiol 2001;12:965-8.

19. Woo S, Chung JW, Hur S, et al. Liver abscess after transarterial chemoembolization in patients with bilioenteric anastomosis: frequency and risk factors. AJR Am J Roentgenol 2013;200:1370-7.

20. Song SY, Chung JW, Han JK, et al. Liver abscess after transcatheter oily chemoembolization for hepatic tumors: incidence, predisposing factors, and clinical outcome. J Vasc Interv Radiol 2001;12:313-20.

21. Carroll BA, Walter JF. Gas in embolized tumors: an alternate hypothesis for its origin. Radiology 1983;147:441-4. 